cases each year. Conclusions: This is the largest case series of RTD to date. Early recognition and prompt riboflavin treatment is essential for survival and optimal outcome.

\section{A.06 \\ Selective amygdalohippocampectomy in pediatric medically refractory temporal lobe epilepsy yields worse seizure outcomes}

CA Elliott (Edmonton)* D Sinclair (Edmonton) A Broad (Edmonton) $K$ Narvacan (Edmonton) T Steve (Edmonton) J Pugh (Edmonton) $T$ Snyder (Edmonton) $B$ Wheatley (Edmonton)

doi: $10.1017 / \operatorname{cjn} .2017 .70$

Background: Selective amygdalohippocampectomy ( $\mathrm{SAH})$ is a surgical option in well-selected cases of pediatric medically refractory temporal lobe epilepsy (TLE). The objective of this study was to compare the surgical outcome and the rate of reoperation for ongoing or recurrent seizures between SAH and anterior temporal lobectomy (ATL) in pediatric TLE. Methods: Retrospective review of 78 pediatric intractable TLE patients referred to the Comprehensive Epilepsy Program at our institution between 1988 and 2015 treated initially with either a trans-middle temporal gyrus SAH (19) or ATL (59). Patients underwent baseline long-term video electroencephalography and 1.5-Tesla MRI. Neuropsychological testing was performed preoperatively and 12-months postoperatively (including reoperations). Results: The mean follow-up was 64 months (range, 12-186 months). The average age at initial surgery was $10.6 \pm 5$ years with an average delay of $5.7 \pm 4$ years between seizure onset and surgery. Ultimately $78 \%$ were seizure-free $(61 / 78)$ at most recent follow-up. Seizure freedom after initial surgical treatment was achieved in $81 \%$ of patients who underwent ATL (48 patients) versus $42 \%$ in SAH ( 8 patients; $\mathrm{p}<0.001)$. Of patients with ongoing disabling seizures following SAH, reoperation (ATL) was offered in 8 resulting in seizure freedom in $63 \%$, without interval neuropsychological decline. Conclusions: SAH amongst well-selected pediatric TLE results in significantly worse seizure control compared with ATL.

\section{A.07}

\section{Genomics of atypical dyskinetic cerebral palsy-opportunities for improved diagnosis and management}

H Goez (Edmonton)* A Matthews (Vancouver) B Al Jabri (Edmonton) I Blydt-Hansen (Vancouver) $J$ Andersen (Edmonton) $M$ Tarailo-Graovac (Vancouver) B Drogemoller (Vancouver) C Shyr (Vancouver) J Lee (Vancouver) A Ghani (Vancouver) G Sinclair (Vancouver) C Ross (Vancouver) W Wasserman (Vancouver) M McKinnon (Vancouver) G Horvath (Vancouver) C Van Karnebeek (Vancouver)

doi: $10.1017 /$ cjn.2017.71

Background: Cerebral palsy (CP) is a debilitating disorder (1). Based on neuromotor impairments it is divided to spastic, dyskinetic and ataxic types (2). Inborn Errors of Metabolism (IEMs), monogenic and chromosomal disorders mimic CP (3). We aimed to identify causal genetic variants in patients with atypical dyskinetic CP in whom known IEMs were ruled out. Timely diagnosis is essential for proper management, especially in conditions that mimic $\mathrm{CP}$ and are treatable. Methods: We enrolled 23 patients with unexplained atypical dyskinetic $\mathrm{CP}$, for whole exome sequencing. Variants were filtered against public and in-house databases to identify variants predicted as damaging (in silico tools and ACMG criteria). We applied a virtual gene panel of known and suspected CP and movement disorder genes and investigated each sample. Results: The participants presented with symptoms including: spasticity, dystonia, choera-athetosis, ataxia and cognitive delays. We identified 23 diagnoses: 13 dominant, 6 recessive and 4 X-linked. 12 patients had movement disorders. In 4 , the diagnoses enabled targeted treatment (neurotransmitter supplements in Unverricht Lundborg diseases (CSTB) and PAK3 deficiency, deep brain stimulation in GNAO1 deficiency, medical diet in Glutaric Aciduria (GCDH). Conclusions: Whole Exome Sequencing contributes to establishing diagnosis in patients with atypical dyskinetic CP resulting in precision medicine and improved health outcomes.

\section{CNS/CSCN Chair's Select Abstracts}

\section{B.01}

Impact of work-hours and sleep on well-being and burnout for physicians-in-training: the prospective RATE Study

D Mendelsohn (Vancouver)* I Despot (Vancouver) PA Gooderham (Vancouver) A Singhal (Vancouver) GJ Redekop (Vancouver) BD Toyota (Vancouver)

doi: $10.1017 / \operatorname{cjn} .2017 .72$

Background: Wearable activity trackers are an innovative tool for measuring sleep and physical activity. The Resident Activity Tracker Evaluation (RATE) is a prospective observational study evaluating the impact of work-hours, sleep, and physical activity on resident well-being, burnout, and job satisfaction. Methods: Residents were recruited from: 1 . general surgery and orthopedics (SURG), 2. internal medicine and neurology (MED) and 3. anesthesia and radiology (RCD). Groups 1 and 2 do not enforce on-call duration restrictions and group 3 had 12-hour restricted-call durations (RCD). Participants wore FitBit activity trackers for 14 days and completed four validated surveys assessing self-reported health, sleepiness, burnout, and job satisfaction. Results: Fifty-nine residents completed the study. 778 days of activity and 244 on-call periods were tracked. Surgical residents worked 24 more hours per week than non-surgical residents (84.3 vs 60.7). Surgical residents had 7 less hours of sleep per week and reported significantly higher Epworth Sleepiness scores. Nearly two-thirds of participants (61\%) scored high burnout on the Maslach depersonalization subscore. Total steps per day and self-reported well-being, burnout, and job satisfaction were comparable between the groups. Conclusions: Despite a positive correlation between work-hours and sleepiness, burnout and well-being were similar among residents. Physical activity did not prevent burnout. These findings are relevant to work-hours policies. 